\title{
A UNIQUENESS THEOREM FOR A BOUNDARY VALUE PROBLEM
}

RIAZ A. USMANI

\begin{abstract}
In this paper it is proved that the two-point boundary value problem, namely $\left(d^{(4)} / d x^{4}+f\right) y=g, y(0)-A_{1}=y(1)-A_{2}=y^{\prime \prime}(0)-$ $B_{1}=y^{\prime \prime}(1)-B_{2}=0$, has a unique solution provided $\inf _{x} f(x)=-\eta>$ $-\pi^{4}$. The given boundary value problem is discretized by a finite difference scheme. This numerical approximation is proved to be a second order convergent process by establishing an error bound using the $L_{2}$-norm of a vector.
\end{abstract}

1. Introduction. Consider the real two-point linear boundary problem

$$
\begin{gathered}
L y \equiv\left[d^{(4)} / d x^{4}+f(x)\right] y=g(x), \quad 0<x<1, \\
y(0)=A_{1}, \quad y(1)=A_{2}, \quad y^{\prime \prime}(0)=B_{1}, \quad y^{\prime \prime}(1)=B_{2},
\end{gathered}
$$

where the functions $f(x)$ and $g(x) \in C[0,1]$. A more general problem of the form

$$
L y=g(x), \quad y(a)=\bar{A}_{1}, \quad y(b)=\bar{A}_{2}, \quad y^{\prime \prime}(a)=\bar{B}_{1}, \quad y^{\prime \prime}(b)=\bar{B}_{2}
$$

can always be transformed into (1) by means of a substitution of the form $X=(x-a) /(b-a)$. Problems of the form (1) frequently occur in plate deflection theory (see Reiss et al. [6]). The analytical solution of (1) is given by Timoshenko and Woinowsky-Krieger [7] provided the functions $f(x)$ and $g(x)$ are constants. In the general case we resort to some numerical techniques. Usmani and Marsden [8] have analyzed a second order convergent finite difference method for (1). Following this, Jain et al. [4] have developed and analysed higher order methods. The problem (1) does not always have a unique solution for all choices of $f(x)$ as is apparent from the example

$$
y^{(4)}-\pi^{4} y=0, \quad y(0)=y(1)=y^{\prime \prime}(0)=y^{\prime \prime}(1)=0
$$

which has as its solution $y(x)=C \sin (\pi x)$ for arbitrary values of $C$. The purpose of this note is to establish a sufficient condition that guarantees a unique solution for (1).

2. A uniqueness theorem. We shall give an elementary proof of the following theorem.

Received by the editors June 6, 1978.

AMS (MOS) subject classifications (1970). Primary 34B05; Secondary 65L10.

Key words and phrases. Brouwer's fixed point theorem, Euclidean and $L_{2}$-norm of a vector, finite difference scheme, local truncation error, plate deflection theory, posteriori error bound, spectral norm of a matrix, two-point boundary value problem.

(C) 1979 American Mathematical Society 0002-9939/79/0000-0557/\$02.75 
THEOREM 1. The boundary value problem (1) has a unique solution provided

$$
\inf _{x} f(x)=-\eta>-\pi^{4} \text {, that is }-f(x)<\eta \text {. }
$$

We preface the proof of this theorem with the following lemmas.

LEMMA 2. If $y(x) \in C^{1}[0,1]$ and $y(0)=y(1)=0$, then

$$
\pi^{2} \int_{0}^{1} y^{2}(x) d x<\int_{0}^{1}\left[y^{\prime}(x)\right]^{2} d x
$$

Let $C[0,1]$ consist of all continuous functions on the interval $I=[0,1]$ and define in this section only $\|y\|=\sup _{x}|y(x)|, x \in I$.

LEMMA 3. If $y(0)=y(1)=0$ and $y(x) \in C[0,1]$, then

$$
\|y\|<0.5\left[\int_{0}^{1}\left[y^{\prime}(x)\right]^{2} d x\right]^{1 / 2} \text {. }
$$

For the proofs of these lemmas the reader should consult Hardy et al. [2, Theorem 256, p. 182] and Lees [5].

LEMMA 4. For the differential system

$$
\begin{gathered}
L y=g(x), \quad y(0)=y(1)=y^{\prime \prime}(0)=y^{\prime \prime}(1)=0, \\
\|y\|<0.5 \pi\|g\| /\left[\pi^{4}-\eta\right] .
\end{gathered}
$$

Proof. The system

$$
\begin{gathered}
\text { (i) } y^{\prime \prime}(x)=z(x), \quad y(0)=y(1)=0, \\
\text { (ii) } z^{\prime \prime}(x)+f(x) y=g(x), \quad z(0)=z(1)=0
\end{gathered}
$$

is equivalent to the differential system of the theorem. On multiplying (3.i) by $y(x)$ and integrating the result from 0 to 1 , we find

$$
-\int_{0}^{1}\left(y^{\prime}\right)^{2} d x=\int_{0}^{1} y z d x
$$

Now using the Cauchy-Schwartz inequality we obtain from the preceding equation

$$
\int_{0}^{1}\left(y^{\prime}\right)^{2} d x<\left[\int_{0}^{1} y^{2} d x\right]^{1 / 2}\left[\int_{0}^{1} z^{2} d x\right]^{1 / 2} .
$$

On using Lemma 2, we derive from the preceding inequality

$$
\left[\int_{0}^{1}\left(y^{\prime}\right)^{2} d x\right]^{1 / 2}<\frac{1}{\pi^{2}}\left[\int_{0}^{1}\left(z^{\prime}\right)^{2} d x\right]^{1 / 2} .
$$

In a similar manner, from (3.ii), we derive

$$
\left[\int_{0}^{1}\left(z^{\prime}\right)^{2} d x\right]^{1 / 2}<\pi^{3} \frac{\|g\|}{\left[\pi^{4}-\eta\right]}
$$


provided $\eta$ satisfies (2). Now from (4) and (5) it follows that

$$
\left[\int_{0}^{1}\left(y^{\prime}\right)^{2} d x\right]^{1 / 2} \leqslant \pi \frac{\|g\|}{\left[\pi^{4}-\eta\right]} .
$$

Lemma 4 now follows from (6) and Lemma 3.

Proof of Theorem 1. Assume that there exist two distinct functions $u(x)$ and $v(x)$ satisfying (1). Then it is easily seen that $\phi(x)=u(x)-v(x)$ satisfies

$$
L \phi=0, \quad \phi(0)=\phi(1)=\phi^{\prime \prime}(0)=\phi^{\prime \prime}(1)=0 .
$$

Now, from Lemma 4 and (7) it follows that $\|\phi\|<0$, which proves $\|\phi\| \equiv 0$ and $u(x) \equiv v(x), x \in I$. This proves that the boundary value problem (1) has at most one solution.

In order to prove that (1) indeed has a solution, we define functions $y_{i}(x)$, $i=1, \ldots, 4$, as solutions of the respective initial value problems.

$$
\begin{array}{llll}
\text { (i) } & L y_{1}=g(x), & y_{1}(0)=A_{1}, & y_{1}^{\prime}(0)=y_{1}^{\prime \prime}(0)=y_{1}^{\prime \prime \prime}(0)=0, \\
\text { (ii) } & L y_{2}=0, & y_{2}^{\prime}(0)=1, & y_{2}(0)=y_{2}^{\prime \prime}(0)=y_{2}^{\prime \prime \prime}(0)=0, \\
\text { (iii) } & L y_{3}=0, & y_{3}^{\prime \prime}(0)=B_{1}, & y_{3}(0)=y_{3}^{\prime}(0)=y_{3}^{\prime \prime \prime}(0)=0, \\
\text { (iv) } & L y_{4}=0, & y_{4}^{\prime \prime \prime}(0)=1, & y_{4}(0)=y_{4}^{\prime}(0)=y_{4}^{\prime \prime}(0)=0 .
\end{array}
$$

From the continuity of $f(x)$ and $g(x)$ we are assured that unique solutions of these initial value problems exist on $[0,1]$. Furthermore the function $z(x) \equiv$ $z(x, s, t)=y_{1}+s y_{2}+y_{3}+t y_{4}, s, t$ being scalars, satisfies the initial value problem

$$
L z=g(x), \quad z(0)=A_{1}, \quad z^{\prime}(0)=s, \quad z^{\prime \prime}(0)=B_{1}, \quad z^{\prime \prime \prime}(0)=t .
$$

The function $z(x)$ will be a solution of (1) provided $s, t$ satisfy

$$
\begin{aligned}
s y_{2}(1)+t y_{4}(1) & =A_{2}-y_{1}(1)-y_{3}(1), \\
s y_{2}^{\prime \prime}(1)+t y_{4}^{\prime \prime}(1) & =B_{2}-y_{1}^{\prime \prime}(1)-y_{3}^{\prime \prime}(1) .
\end{aligned}
$$

If $\Delta=y_{2}(1) y_{4}^{\prime \prime}(1)-y_{2}^{\prime \prime}(1) y_{4}(1) \neq 0$, a unique solution of the preceding linear system can be found, say $s^{*}, t^{*}$, and the corresponding function $z\left(s, s^{*}, t^{*}\right)$ then is the unique solution of (1). However, if $\Delta=0$, then

$$
y_{2}(1) / y_{2}^{\prime \prime}(1)=y_{4}(1) / y_{4}^{\prime \prime}(1)=p \text { (constant). }
$$

We can assume that $p \neq 0$, because if $p=0$, then $y_{2}(1)=0$ and the solution of

$$
L y_{2}=0, \quad y_{2}(0)=y_{2}^{\prime \prime}(0)=y_{2}^{\prime \prime \prime}(0)=y_{2}(1)=0
$$

from Taylor series has the property that $y_{2}^{\prime}(0)=0$, contradicting the original assumption that $y_{2}^{\prime}(0)=1$. Similarly $p$ cannot be unbounded. Thus it follows that $y_{2}(1)=p y_{2}^{\prime \prime}(1), p<\infty$.

Now using the system (8.ii), and the Taylor series, we obtain

$$
\begin{array}{ll}
y_{2}(1)=1-\frac{1}{24} f(\alpha) y_{2}(\alpha), & 0<\alpha<1, \\
y_{2}^{\prime \prime}(1)=-0.5 f(\beta) y_{2}(\beta), & 0<\beta<1 .
\end{array}
$$


On combining $y_{2}(1)=p y_{2}^{\prime \prime}(1)$ with equations (9) we obtain

$$
f(\alpha) y_{2}(\alpha)-12 p f(\beta) y_{2}(\beta)=24,
$$

for all $f(x) \in C$. In an attempt to determine $y_{2}(\alpha)$ and $y_{2}(\beta)$, we choose $f(x) \equiv 1$ and $f(x) \equiv-1$, giving the system

$$
y_{2}(\alpha)-12 p y_{2}(\beta)=24, \quad-y_{2}(\alpha)+12 p y_{2}(\beta)=24 \text {. }
$$

But this latter system in the unknowns $y_{2}(\alpha)$ and $y_{2}(\beta)$ is inconsistent. We thus conclude that $\Delta$ cannot vanish and the proof of the Theorem 1 is completed.

3. A discrete boundary value problem. Let $N$ be a positive integer and $h=(N+1)^{-1}$. We define the grid points $x_{n}=a+n h, n \in\{0, N+1\} \cup S$ where $S=(1,2, \ldots, N)$. We denote by $\Phi$ the set of all real-valued functions defined on $\left\{x_{n}\right\}, n \in S$. Clearly $\Phi$ is a real linear space of dimension $N$. Also let $\|u\|=\left[\Sigma_{i} h u_{i}^{2}\right]^{1 / 2}$, where $u_{i} \equiv u\left(x_{i}\right)$. Note that $\|\cdot\|$ defines the $L_{2}$-norm of a vector, a natural definition of a norm on vectors since this norm converges to $\left[\int_{0}^{1} u^{2}(x) d x\right]^{1 / 2}$ as $h \rightarrow 0$. We also have $\|u\|=\sqrt{h}\|u\|_{2}$ where $\|\cdot\|_{2}$ is the Euclidean norm (see Isaacson and Keller [3]). For a given matrix $A=\left(a_{i j}\right)$, the matrix norm induced by the Euclidean vector norm we define the Hilbert or spectral norm of a matrix by $\|A\|_{2}=\sqrt{\mu}$ where $\mu$ is the largest eigenvalue of $A^{*} A$. Here the operation * denotes the conjugate transpose of a matrix.

We now discretize the problem (1) by the following finite difference scheme

$$
\begin{array}{r}
\delta^{4} y\left(x_{n}\right)=h^{4} y^{(4)}\left(x_{n}\right)+\frac{1}{6} h^{6} y^{(6)}\left(\omega_{n}\right), \quad n=2, \ldots, N-1, \\
x_{n-2}<\omega_{n}<x_{n+2},
\end{array}
$$

$$
\begin{aligned}
y\left(x_{N-2}\right) & -4 y\left(x_{N-1}\right)+6 y\left(x_{N}\right)-2 y\left(x_{N+1}\right) \\
= & -h^{2} y^{\prime \prime}\left(x_{N+1}\right)+h^{4}\left[y^{(4)}\left(x_{N}\right)-\frac{1}{12} h^{4} y^{(4)}\left(x_{N+1}\right)\right]+t_{N},
\end{aligned}
$$

where $t_{i}=\frac{59}{360} h^{6} y^{(6)}\left(\omega_{i}\right), i=1, N, x_{0}<\omega_{1}<x_{3}, x_{N-2}<\omega_{N}<x_{N+1}$. Set $Y=$ $\left(y_{n}\right)$ where $y_{n}$ is an approximation to $y\left(x_{n}\right), y(x)$ being the exact solution of (1). As in [4], [8], we obtain, on neglecting the local truncation errors $t_{n}$, noting $y^{(4)}=-f(x) y+g(x)$ and $y\left(x_{n}\right) \simeq y_{n}$,

$$
P^{2} Y=-h^{4} D Y+C, \quad P^{-1}>0,
$$

(see [8]) where the tridiagonal matrix $P=\left(p_{i j}\right)$ is given by $p_{i i}=2, p_{i j}=-1$ for $|i-j|=1$, otherwise $p_{i j}=0 ; D=\operatorname{diag}\left(f_{n}\right)$ is a diagonal matrix and the column vector $C$ depends on $g(x)$ and the boundary conditions. The matrix $P$ is symmetric and positive definite and it is known that its eigenvalues are $4 \sin ^{2}(m \pi h / 2), m \in S$. Thus the eigenvalues of $P^{2}$ are

$$
\lambda_{m}=16 \sin ^{4}(m \pi h / 2), \quad m \in S
$$


LEMMA 5. $\pi^{4} h^{4}\left(1-\pi^{2} h^{2} / 6\right) \leqslant \lambda_{1} \leqslant \pi^{4} h^{4}$.

The inequality follows from $\theta-\theta^{3} / 6 \leqslant \sin \theta<\theta$ for $0<\theta<\pi / 2$ and $(1-x)^{n}>1-n x$ for small values of $x$. Also the eigenvalues satisfy

$$
0<\lambda_{1}<\lambda_{2}<\cdots<\lambda_{N}
$$

Since $P$ is symmetric, it is easy to see that

$$
\left\|P^{-2}\right\|_{2}=1 / \lambda_{1} \text {. }
$$

Lemma 6. Assume that $f(x)$ satisfies (2) and that $h_{0}$ is such that

$$
\eta<\pi^{4}\left(1-\pi^{2} h_{0} / 6\right) .
$$

Furthermore if $h<h_{0}$, and $u, v \in \Phi$ satisfy

$$
P^{2} u=-h^{4} D u+C_{1}, \quad P^{2} v=-h^{4} D v+C_{2},
$$

then $\|u-v\| \leqslant K\left(h_{0}\right)\left\|C_{1}-C_{2}\right\|$, where

$$
K\left(h_{0}\right)=h^{-4}\left[\pi^{4}\left(1-\pi^{2} h_{0}^{2} / 6\right)-\eta\right]^{-1} .
$$

Proof. From the hypothesis it follows

$$
\begin{aligned}
P^{2}(u-v) & =-h^{4} D(u-v)+\left(C_{1}-C_{2}\right), \\
(u-v) & =P^{-2}\left[-h^{4} D(u-v)+\left(C_{1}-C_{2}\right)\right], \\
\|u-v\|_{2} & \leqslant\left(1 / \lambda_{1}\right)\left[\eta h^{4}\|u-v\|_{2}+\left\|C_{1}-C_{2}\right\|_{2}\right],
\end{aligned}
$$

by (2) and (14) or

$$
\left(\lambda_{1}-\eta h^{4}\right)\|u-v\| \leqslant\left\|C_{1}-C_{2}\right\| .
$$

Now on using Lemma 5 and (15), the result of Lemma 6 follows.

REMARK. If $\eta=0$, the constant $h_{0}<0.77$.

LEMMA 7. If $f(x)$ satisfies (2) and if $Y$ is a solution of (11), then

$$
\|Y\| \leqslant K\left(h_{0}\right) \cdot\|C\| \text {. }
$$

Proof. Put $u=Y, C_{1}=C, v=C_{2}=0$ in Lemma 6, then (17) follows.

THEOREM 2. If $f(x)$ satisfies (2), then the discrete boundary value problem (11) has a unique solution.

Proof. Clearly, Lemma 6 implies that (11) has at most one solution. Let $\Omega=\left\{u \in \Phi:\|u\| \leqslant K\left(h_{0}\right)\|C\|\right\}$. Define a mapping $T: u \rightarrow v$ by means of the relation

$$
P^{2} v=-h^{4} D u+C .
$$

Since $P^{-1}>0$, it follows that (18) has exactly one solution for a given $u$. 
Consider $T u=v$ and use (18) to deduce

$$
\begin{aligned}
\|v\| & <\left[h^{4} \eta\|u\|+\|C\|\right] / \lambda_{1} \\
& <\left[\left(h^{4} \eta K\left(h_{0}\right)+1\right)\|C\|\right] /\left[\pi^{4} h^{4}\left(1-\pi^{2} h_{0}^{2} / 6\right)\right] \\
& <K\left(h_{0}\right)\|C\|,
\end{aligned}
$$

on using (16). This proves that $T$ maps $\Omega$ into itself. Let $\varepsilon>0$ be given, we can choose $\delta(\varepsilon)$

$$
\delta=\left[\varepsilon \pi^{4}\left(1-\pi^{2} h_{0}^{2} / 6\right)\right] / \eta, \quad \eta \neq 0 .
$$

Now if $T u_{1}=y_{1}, T u_{2}=y_{2}$, then

$$
\begin{aligned}
\left\|T u_{1}-T u_{2}\right\| & =\left\|y_{1}-y_{2}\right\| \\
& =\left\|P^{-2}\left(-h^{4} D u_{1}+C\right)-P^{-2}\left(-h^{4} D u_{2}+C\right)\right\| \\
& <h^{4} \eta\left\|u_{1}-u_{2}\right\| / \lambda_{1}<\varepsilon,
\end{aligned}
$$

provided $\left\|u_{1}-u_{2}\right\|<\delta$ given by (19) and $\lambda_{1}>\pi^{4} h^{4}\left(1-\pi^{2} h_{0}^{2} / 6\right)$. This shows that $T$ is continuous on $\Omega$. Hence, by Brouwer's fixed point theorem [1], there is a $u \in \Omega$ such that $T u=u$, and this is clearly a solution of (18) and hence of (11). This completes the proof of the theorem.

Note. For $\eta=0$, an obvious modification of the argument still proves the preceding theorem.

4. An approximation theorem. In this concluding section we establish an a posteriori bound. We note that the system of linear equations based on (10) can be written as

$$
P^{2} \tilde{Y}=-h^{4} D \tilde{Y}+C+T
$$

where $\tilde{Y}=\left(y\left(x_{n}\right)\right) \in \Phi$ and clearly

$$
\|T\| \leqslant \frac{1}{6} h^{6} M_{6}
$$

where $M_{6}=\max _{x}\left|d^{(6)} y / d x^{6}\right|, 0<x \leqslant 1$. If we subtract (11) from (20), we obtain an error equation, namely

$$
P^{2} E=-h^{4} D E+T
$$

where $E=\left(e_{n}\right) \in \Phi$ and $e_{n}=y\left(x_{n}\right)-y_{n}$.

THEOREM 3. If $f(x)$ satisfies (2), then for $h<h_{0}$ :

$$
\|E\|=O\left(h^{2}\right) \text {. }
$$

Proof. From Lemma 7, it follows that

$$
\|E\| \leqslant K\left(h_{0}\right)\|T\|=O\left(h^{2}\right)
$$

using (16) and (22). In fact

$$
\|E\| \leqslant \frac{1}{6} M_{6} h^{2}\left[\pi^{4}\left(1-\pi^{2} h_{0}^{2} / 6\right)-\eta\right]^{-1} .
$$

ACKNOWLEDGEMENT. The author acknowledges the National Research Council of Canada's financial support. 


\section{REFERENCES}

1. N. Dunford and J. T. Schwartz, Linear operators. I. General theory, Interscience, New York, 1958.

2. G. H. Hardy, J. E. Littlewood and G. Polya, Inequalities, Cambridge Univ. Press, London and New York, 1952.

3. E. Isaacson and H. B. Keller, Analysis of numerical methods, Wiley, New York, 1966.

4. M. K. Jain, S. R. K. Iyengar and J. S. V. Saldanha, Numerical integration of a fourth order ordinary differential equation, J. Engrg. Math. 11 (1977), 373-380.

5. M. Lees, Discrete methods for nonlinear two-point boundary value problems, Numerical Solutions of Partial Differential Equations (Proc. Sympos. Univ. Maryland, 1965), Academic Press, New York, 1966, pp. 59-72.

6. E. L. Reiss, A. J. Callegari and D. S. Ahluwalia, Ordinary differential equations with applications, Holt, Rinehart and Winston, New York, 1976.

7. S. Timoshenko and S. Woinowsky-Krieger, Theory of plates and shells, McGraw-Hill, New York, 1959.

8. R. A. Usmani and M. J. Marsden, Numerical solution of some ordinary differential equations occurring in plate deflection theory, J. Engrg. Math. 9 (1975), 1-10.

Department of Mathematics, University of Manitoba, Winnipeg, Manitoba, Canada R3T 2N2 